\title{
A Model Applied to Surface Tension of Liquids
}

\author{
D.V. Singh ${ }^{1}$, A. Kumar ${ }^{2}$, R.Kumari ${ }^{3}$ and N.Dass ${ }^{3 *}$ \\ ${ }^{1}$ Physics Department, College of Engineering Roorkee, Roorkee, India \\ ${ }^{2}$ Physics Department, Gurukula Kangri Vishwavidyalaya, Haridwar, India \\ ${ }^{3}$ Physics Department, M S Post Graduate College, Saharanpur, India \\ "Corresponding Author: ndass2@ rediffmail.com
}

Available online at: www.isroset.org

Accepted: 27/Jun/2018, Online: 31/Aug/2018

\begin{abstract}
In the present paper, a model is suggested to study the temperature dependence of surface tension in liquids and is applied in more than sixty liquids including liquid metals, rare gas liquids, inorganic and organic liquids. A very good agreement is found between the experimental values and the computed results in case of each liquid.
\end{abstract}

Keywords- Surface tension, temperature, liquid metals and liquefied gases, organic and inorganic liquids

\section{INTRODUCTION}

Surface tension of liquid is an important property and has wide applications in understanding the phenomenon of (i) wettability (ii) migration (iii) atomic bonding and (iv)two-phase heat transfer along with the correlation with other thermodynamic properties. Further, the surface tension is found to have the practical applications in material science, chemical engineering and environmental science besides physics, chemistry and petroleum engineering. However, the surface tension of liquid is found to decrease with rise in temperature and vanishes at critical temperature. Under these circumstances, a study of temperature dependence of surface tension of liquids become quite important and particularly in the development of a model for the liquid state with the help of physical thermodynamic properties.

In literature, many empirical and semi-empirical relations [1-14] are available to represent the temperature dependence of surface tension of liquids. All these relations make use of reduced parameters which include the critical temperature or some other property of the concerned liquid and hence adjustable parameters required for the study of temperature dependence of surface tension in the liquids become quite large. Further, these relations have limited success in the sense that these relations could not be applied in case of those liquids whose critical temperature is not known. Hence, there is a need of a model/relation between surface tension and temperature which can be applied to different types of liquids and that too for the widest possible temperature range even without knowing the value of the critical temperature or some other physical property of the concerned liquid.
Therefore, the aim of the present paper is to suggest a model/relation between surface tension of liquid and the temperature .The model/relation can be further used to obtain thermo-physical properties like para-chore, surface entropy and surface enthalpy.

\section{THEORY}

To study the temperature dependence of surface tension in liquids, it is suggested that the ratio of second to first temperature derivative of surface tension is a constant and is expressed as

$$
\left(\frac{d^{2} \sigma}{d \mathrm{~T}^{2}}\right) /\left(\frac{d \sigma}{d \mathrm{~T}}\right)=-\mathrm{Z}
$$

Successive integration of eq.(1) with respect to temperature in limits $\mathrm{T}=\mathrm{T}$ and $\mathrm{T}=\mathrm{T}_{0}$ gives the following relations:

$$
\begin{aligned}
& \frac{d \sigma}{d \mathrm{~T}}=\sigma^{\prime}\left(\mathrm{T}_{0}\right) \exp \left[-\mathrm{Z}\left(\mathrm{T}-\mathrm{T}_{0}\right)\right] \\
& \text { and } \\
& \sigma(\mathrm{T})=\sigma\left(\mathrm{T}_{0}\right)+\frac{\sigma^{\prime}\left(\mathrm{T}_{0}\right)}{\mathrm{Z}}\left[1-\exp \left\{-\mathrm{Z}\left(\mathrm{T}-\mathrm{T}_{0}\right)\right\}\right]
\end{aligned}
$$

In eqs.(1)-(3). $\sigma\left(\mathrm{T}_{0}\right)$ and $\sigma^{\prime}\left(\mathrm{T}_{0}\right)$ represent surface tension and its first temperature derivative at $\mathrm{T}=\mathrm{T}_{0}$ where 
$\mathrm{T}_{0}$ is the reference temperature. $\mathrm{Z}$ is a temperature independent constant.

\section{RESULTS AND DISCUSSIONS}

The relation given by eq.(3) is applied in liquid metals, rare gas liquids, inorganic and organic liquids including ionic liquids. In total, we have applied eq.(3) in more than sixty liquids. The results are reported in table 1 . Table1 includes the temperature range studied, the values of constants $\sigma^{\prime}\left(\mathrm{T}_{0}\right)$ and $\mathrm{Z}$ along with root mean square deviation (RMSD) in case of each liquid studied here. RMSD is a parameter which represents the goodness of fit. The values of the constants are determined by least square fit.

The surface thermodynamic properties like surface entropy and surface enthalpy can be estimated once the temperature dependence of surface tension is known. The surface entropy, $S^{\sigma}$, and surface enthalpy, $H^{\sigma}$, can be computed from the relations:

$$
S^{\sigma}=-\left(\frac{d \sigma}{d \mathrm{~T}}\right)
$$

$$
H^{\sigma}=\sigma-\mathrm{T}\left(\frac{d \sigma}{d \mathrm{~T}}\right)
$$

(5)

It is evident that $\sigma^{\prime}\left(\mathrm{T}_{0}\right)$ expresses the value of surface entropy at temperature $\mathrm{T}_{0}$. With the help of eq.(2), one can easily calculate the values of surface entropy and surface enthalpy as a function of temperature.

\section{CONCLUSION}

Following important points emerges out from the present study:

(a) It is evident from table 1 that the present relation is quite successful and valid in representing the temperature dependence of surface tension in all the liquids studied here.

(b) From the present relation, one can easily obtain surface thermodynamic properties like surface entropy and surface enthalpy. To save space, these properties are not reported in the present paper.

(c) The present relation is free from critical temperature and other physical properties of the liquid concerned.

(d) The present model has only two adjustable parameters and one of the parameter represents the surface entropy. (e) The relation is useful in the interpolation as well as in the extrapolation of surface tension data at any given temperature.

\section{REFERENCES}

[1] N.Auerbach, Experientia, vol.4, pp.473, 1948.

[2] K. Alterburg, Z.Phys.Chem., pp.145-147, 1950

[3] J. R. Brock and R B Bird, AICHE journal, vol.1, pp.174-177, 1955.

[4] D. D Patterson and A. K. Rastogi, J Phys. Chem., vol.74, pp.10671071,1970

[5] D. I. Hakin, D. Steinberg and L I Stiel, Ind.Eng.Chem.Funda., vol.10, pp.174, 1971.

[6] J. A. Hugill and A J Van Welsenes, Fluid Phase Equil., vol.29, pp.383, 1986

[7] R. P. Chhabra, High Temp-High Press., vol.21,pp.171-174, 1990.

[8] H.M.Lu and Q. Jiang, J.Phys.Chem.B, vol.109, pp.15463-15468, 2005.

[9] K. S. Pitzer Thermodynamics, $3^{\text {rd }}$ ed. Mcgraw-Hill, Nework, 1995.

[10] Y.X.Zuo and E. H. Stenley, Can. J. Chem. Eng., Vol.75,pp.1130$1132,1997$.

[11] C. Mrqueu et al, Fluid Phase Equil, Vol. 172, pp.169-171, 2000.

[12] S. T. Bowden, J Chem Phys., vol.23,pp.2454, 1955.

[13] N. K. Sanyal and S S Mitra, J.Chem Phys., vol.24, pp.473474, 1956.

[14] N. Dass and O. Singh, J Phy. Soc Japan, vol 28, pp.806-807, 1970

[15] V.W. Lazarev, Teoreticheskaya i Eksperimental'naya Khimiya,vol.3,pp.504-507, 1967.

[16] F. Aqra and A. Ayyad, Phys and Chem Liquids, vol.50, pp.336345,2012

[17] M. H. Ghatee and A. Boushehri, High Temp- High Pressures, vol.26, pp507-514, 1994.

[18] S. Kim and S. Chang, Daehan Hwahak Hwoejee, vol.9, pp.110114, 1965.

[19] J. Amoros, J, R, Solana and E. Villar, Materials Science, vol.7, pp.127-136, 1982

[20] L. Lomba et. al. J. Chem. Thermodynamics, vol.65, pp. 34-41, 2013

[21] M. H. Ghatee et. al., J. Chen. Engg. Data , vol.57, 2095-2101, 2012.

[22] P. Jianxin and L. Yigang, Phys. and Chem Liquids, vol.47,pp.267-273, 2009.

[23] W. Gao, X. Zhao and Z. Liu, J. Chem. Engg. Data, vol.54, pp.1761-1763, 2009.

[24] J. Klomfar, M. Souckova and J. Patak, J. Chem. Engg. Data, vol.59, pp. 2263-2274, 2014.

[25] T. M. Koller et. al.; J. Chem. Engg. Data, vol.60,pp. 2665-2673, 2015.

[26] G. Vazquez, E. Alvarez and M. Navaza, J. Chem. Engg. Data, vol.40, pp. 611-614, 1995.

[27] L. G. Sanchez et. al., J. Chem. Engg. Data, vol.54, pp. $2803-$ 2812,2009 .

[28] H. F. D. Almeida et. al. J. Chem. Thermodynamics, vol. 57, pp.372-379, 2013

[29] E. M. Fendu and F. Oprea, J. Chem. Engg. Data, vol.58, pp.2898$2903,2013$.

[30] J. Vijande et. al. J. Chem. Engg. Data, vol.51,pp. 1778-1782, 2006.

[31] P. K. Chhotaray and R.L. Gardas, J. Chem. Engg. Data, vol.60,pp. 1868- 1877, 2015. 
[32] L. Lomba et. al. J. Chem. Engg. Data, vol. 59, pp. 329-338, 2014.

[33] L. Lomba et. al. J. Chem. Engg. Data, vol. 58, pp.1193-1197, 2013.
[34] S. Halas and T. Durakiewicz, J. Phys.: Condens. Matter, vol. 14, pp.L735-L737, 2002.

[35] IAWPS Release on Surface Tension of Ordinary Water, September, 1994.

[36] I. C. Sanchez, J. Chem. Phys., vol79, pp. 405-407, 1983.

Table 1- Input parameters and RMSD in case of different liquids

\begin{tabular}{|c|c|c|c|c|c|c|}
\hline S. No. & Liquid & $\begin{array}{l}\text { Temp } \\
\text { Range (K) }\end{array}$ & $\begin{array}{l}\sigma^{\prime}\left(\mathrm{T}_{0}\right) \\
\left(\mathbf{m N m ^ { - 1 }}\right. \\
\left.\mathbf{K}^{-1}\right)\end{array}$ & $\mathbf{Z}\left(\mathbf{K}^{-1}\right)$ & $\begin{array}{l}\text { RMSD } \\
\left(\mathbf{m N m}^{-1}\right)\end{array}$ & Ref. \\
\hline 1 & $\mathrm{Sn}$ & $250-1700$ & 0.0738 & 0.0001 & 2.07 & 15 \\
\hline 2 & In & $200-1700$ & 0.1194 & 0.0003 & 3.68 & 15 \\
\hline 3 & $\mathrm{~Pb}$ & $330-1400$ & 0.1246 & 0.0001 & 2.91 & 15 \\
\hline 4 & $\mathrm{Tl}$ & $310-1100$ & 0.0834 & 0.0001 & 1.35 & 15 \\
\hline 5 & $\mathrm{Bi}$ & $300-1300$ & 0.0814 & 0.0001 & 1.58 & 15 \\
\hline 6 & $\mathrm{Cd}$ & $325-600$ & 0.2085 & -0.0001 & 0.93 & 15 \\
\hline 7 & Ge & $950-1700$ & 0.0976 & 0.0001 & 2.38 & 15 \\
\hline 8 & $\mathrm{Si}$ & $1687-1825$ & 0.2968 & -0.0003 & 0.11 & 16 \\
\hline 9 & $\mathrm{~K}$ & 336.4-1032.2 & 0.0066 & -0.0001 & 0.003 & 17 \\
\hline 10 & $\mathrm{Li}$ & $453.7-1645$ & 01410 & 0.0001 & 0.012 & 17 \\
\hline 11 & $\mathrm{Na}$ & $371.0-1151.2$ & 0.0010 & 0.0001 & 0.002 & 17 \\
\hline 12 & $\mathrm{Rb}$ & $312.7-959.2$ & 0.0058 & 0.0002 & 0.003 & 17 \\
\hline 13 & Cs & $301.6-943.0$ & 0.0048 & -0.0001 & 0.003 & 17 \\
\hline 14 & I & $398.15-428.5$ & 0.1110 & 0.0105 & 0.033 & 18 \\
\hline 15 & $\mathrm{Ar}$ & $83.81-140.0$ & 0.2642 & -0.0072 & 0.039 & 19 \\
\hline 16 & $\mathrm{Xe}$ & $170.0-280.0$ & 0.1970 & -0.0046 & 0.084 & 19 \\
\hline 17 & $\mathrm{Ne}$ & $24.55-30.0$ & 0.3574 & -0.0031 & 0.013 & 19 \\
\hline 18 & $\mathrm{Kr}$ & $115.79-119.04$ & 0.2091 & 0.0543 & 0.026 & 19 \\
\hline 19 & Methyl Levulinate & $278.15-338.15$ & 0.1111 & -0.0001 & 0.034 & 20 \\
\hline 20 & 2-HEAF & $298.15-353.15$ & 0.0731 & 0.0003 & 0.027 & 21 \\
\hline 21 & $\mathrm{CO}_{2}$ & $221.0-298.20$ & 0.3122 & -0.0115 & 0.142 & 22 \\
\hline 22 & 2,2Dimethylbutane & $233.08-378.23$ & 0.1083 & 0.0011 & 0.067 & 23 \\
\hline 23 & {$[\mathrm{THTDP}]\left[\mathrm{NTf}_{2}\right]$} & 273.49-364.76 & 0.0541 & -0.0021 & 0.068 & 24 \\
\hline 24 & [THTDP][DCA] & $283.61-365.21$ & 0.0725 & -0.0027 & 0.080 & 24 \\
\hline 25 & {$[\mathrm{EMIM}]\left[\mathrm{C}(\mathrm{CN})_{3}\right]$} & $283.18-353.36$ & 0.0714 & -0.0061 & 0.144 & 25 \\
\hline 26 & {$[\mathrm{BMIM}]\left[\mathrm{C}(\mathrm{CN})_{3}\right]$} & 283.39-353.37 & 0.1517 & 0.0136 & 0.143 & 25 \\
\hline 27 & {$[\mathrm{HMIM}]\left[\mathrm{C}(\mathrm{CN})_{3}\right]$} & $283.23-353.53$ & 0.0756 & 0.0001 & 0.075 & 25 \\
\hline 28 & {$[\mathrm{OMIM}]\left[\mathrm{C}(\mathrm{CN})_{3}\right]$} & $283.28-353.03$ & 0.0906 & 0.0054 & 0.088 & 25 \\
\hline 29 & {$[\mathrm{EMIM}]\left[\mathrm{B}(\mathrm{CN})_{4}\right]$} & $283.10-353.45$ & 0.1137 & 0.0070 & 0.081 & 25 \\
\hline 30 & {$[\mathrm{BMIM}]\left[\mathrm{B}(\mathrm{CN})_{4}\right]$} & $283.23-353.30$ & 0.0825 & -0.0022 & 0.111 & 25 \\
\hline 31 & {$[\mathrm{HMIM}]\left[\mathrm{B}(\mathrm{CN})_{4}\right]$} & $283.27-353.27$ & 0.0811 & -0.0020 & 0.101 & 25 \\
\hline 32 & {$[\mathrm{OMIM}]\left[\mathrm{B}(\mathrm{CN})_{4}\right]$} & $283.23-353.28$ & 0.0824 & -0.0022 & 0.112 & 25 \\
\hline 33 & {$[\mathrm{DMIM}]\left[\mathrm{B}(\mathrm{CN})_{4}\right]$} & 283.19-353.24 & 0.0866 & 0.0027 & 0.091 & 25 \\
\hline 34 & 1-Propanol & 293.15-323.15 & 0.0794 & -0.0001 & 0.009 & 26 \\
\hline 35 & 2-Propanol & $293.15-323.15$ & 0.1012 & -0.0001 & 0.008 & 26 \\
\hline 36 & Methanol & $293.15-323.15$ & 0.0949 & 0.0022 & 0.025 & 26 \\
\hline 37 & Ethanol & $293.15-323.15$ & 0.0909 & 0.0065 & 0.022 & 26 \\
\hline 38 & {$[\mathrm{BMIM}] \mathrm{MeSO}_{4}$} & $294.55-353.15$ & 0.0235 & -0.0371 & 0.601 & 27 \\
\hline 39 & {$\left[\mathrm{p}_{666}(14)\right]\left[\mathrm{NTf}_{2}\right]$} & $298.20-343.20$ & 0.0744 & 0.0074 & 0.050 & 28 \\
\hline 40 & {$\left[\mathrm{p}_{666}(14)\right][\mathrm{Deca}]$} & $298.00-343.00$ & 0.0541 & -00057 & 0.034 & 28 \\
\hline 41 & {$\left[\mathrm{p}_{666}(14)\right][\mathrm{phosh}]$} & $297.90-342.90$ & 0.0592 & -0.0073 & 0.035 & 28 \\
\hline 42 & {$\left[\mathrm{p}_{666}(14)\right]\left[\mathrm{N}(\mathrm{CN})_{2}\right]$} & $298.40-343.40$ & 0.0755 & 0.0078 & 0.025 & 28 \\
\hline 43 & {$\left[\mathrm{p}_{666}(14)\right][\mathrm{Br}]$} & $289.20-343.40$ & 0.0712 & 0.0022 & 0.060 & 28 \\
\hline 44 & {$\left[\mathrm{p}_{666}(14)\right][\mathrm{Cl}]$} & $298.60-343.30$ & 0.0620 & -0.0055 & 0.050 & 28 \\
\hline 45 & {$\left[\mathrm{p}_{666}(14)\right]\left[\mathrm{CH}_{3} \mathrm{SO}_{3}\right.$} & $298.30-343.50$ & 0.0736 & 0.0030 & 0.028 & 28 \\
\hline
\end{tabular}




\begin{tabular}{|l|l|l|l|l|l|l|}
\hline 46 & Tetrapropylene Glycol & $298.15-468.15$ & 0.0823 & -0.0001 & 0.035 & 29 \\
47 & 1-Butanol & $288.15-308.15$ & 0.0848 & 0.0098 & 0.022 & 30 \\
48 & 1-Octanol & $288.15-308.15$ & 0.0848 & 0.0098 & 0.022 & 30 \\
49 & 1-Rexanol & $288.15-308.15$ & 0.0848 & 0.0098 & 0.022 & 30 \\
50 & PAAC & $293.15-333.15$ & 0.0858 & -0.0019 & 0.022 & 31 \\
51 & 3 HPAF & $293.15-333.15$ & 0.1017 & -0.0200 & 0.020 & 31 \\
52 & 3 HPATFac & $293.15-333.15$ & 0.0831 & -0.0001 & 0.026 & 31 \\
53 & 5-Methylfurfural & $278.15-338.15$ & 0.1210 & 0.0007 & 0.030 & 32 \\
54 & Tetrahydrofurfuryl & $288.15-338.15$ & 0.1013 & 0.0001 & 0.003 & 32 \\
& Alcohol & & & & & \\
55 & Methyl Acrylate & $278.15-338.15$ & 0.1350 & 0.0017 & 0.035 & 33 \\
56 & Ethyl Acrylate & $278.15-338.15$ & 0.11920 & 0.0001 & 0.028 & 33 \\
57 & Butyl Acrylate & $278.15-338.15$ & .1085 & -0.0005 & 0.080 & 33 \\
58 & Mercury & $273.15-523.15$ & 0.1548 & -0.0027 & 0.070 & 34 \\
59 & Water & $273.15-423.15$ & 0.1460 & -0.0029 & 0.040 & 35 \\
60 & Heptane & $273.15-523.15$ & 0.1056 & 0.0017 & 0.113 & 36 \\
61 & Benzene & $278.65-473.15$ & 0.1364 & 0.0013 & 0.049 & 36 \\
& & & & & & \\
\hline
\end{tabular}

\section{Author Profile}

Mr. N. Dass obtained B.Sc and M.Sc from Birla Science College, Pilani (India) in year 1960 and 1962. He obtained his Ph.D from University of Roorkee (now IIT) in year 1969. He has published more than seventy research papers in International and National Journals. Thirty papers have been accepted for presentation in various International Conferences. He has been the reviewer to some International Journals. After retiring from University of Roorkee, he is presently working in College of Engineering Roorkee, Roorkee (India). 